\title{
IR action spectroscopy of glycosaminoglycan oligosaccharides
}

\author{
Maike Lettow ${ }^{1,2} \cdot$ Márkó Grabarics $^{1,2} \cdot$ Eike Mucha ${ }^{1} \cdot$ Daniel A. Thomas ${ }^{1} \cdot$ Łukasz Polewski $^{1,2} \cdot$ Joanna Freyse $^{3}$. \\ Jörg Rademann ${ }^{3} \cdot$ Gerard Meijer $^{1} \cdot$ Gert von Helden ${ }^{1} \cdot$ Kevin Pagel $^{1,2}$ (B]
}

Received: 8 October 2019 / Revised: 4 November 2019 / Accepted: 11 November 2019 / Published online: 18 December 2019

(C) The Author(s) 2019

\begin{abstract}
Glycosaminoglycans (GAGs) are a physio- and pharmacologically highly relevant class of complex saccharides, possessing a linear sequence and strongly acidic character. Their repetitive linear core makes them seem structurally simple at first glance, yet differences in sulfation and epimerization lead to an enormous structural diversity with only a few GAGs having been successfully characterized to date. Recent infrared action spectroscopic experiments on sulfated mono- and disaccharide ions show great promise. Here, we assess the potential of two types of gas-phase action spectroscopy approaches in the range from 1000 to $1800 \mathrm{~cm}^{-1}$ for the structural analysis of complex GAG oligosaccharides. Synthetic tetra- and pentasaccharides were chosen as model compounds for this benchmark study. Utilizing infrared multiple photon dissociation action spectroscopy at room temperature, diagnostic bands are largely unresolved. In contrast, cryogenic infrared action spectroscopy of ions trapped in helium nanodroplets yields resolved infrared spectra with diagnostic features for monosaccharide composition and sulfation pattern. The analysis of GAGs could therefore significantly benefit from expanding the conventional MS-based toolkit with gas-phase cryogenic IR spectroscopy.
\end{abstract}

Keywords Glycosaminoglycans · Fondaparinux · Mass spectrometry $\cdot$ Action spectroscopy $\cdot$ Cryogenic infrared spectroscopy

\section{Introduction}

Living systems encode information and function in the sequence of biopolymers. Determining the primary structure of nucleic acids and proteins has played a central role in the progress in life sciences, with an arsenal of sensitive and automated sequencing strategies currently available. The high-

Parts of this work were presented at ANAKON 2019, Münster (Germany) in March 2019, and have been awarded with an ABC Best Poster Award.

Maike Lettow and Márkó Grabarics contributed equally to this work.

Electronic supplementary material The online version of this article (https://doi.org/10.1007/s00216-019-02327-7) contains supplementary material, which is available to authorized users.

Kevin Pagel

kevin.pagel@fu-berlin.de

1 Department of Molecular Physics, Fritz Haber Institute of the Max Planck Society, Faradayweg 4-6, 14195 Berlin, Germany

2 Institute of Chemistry and Biochemistry, Freie Universität Berlin, Takustraße 3, 14195 Berlin, Germany

3 Institute of Pharmacy, Medicinal Chemistry, Freie Universität Berlin, Königin-Luise-Str. 2+4, 14195 Berlin, Germany throughput de novo sequencing of glycans, however, is still one of the biggest challenges in bioanalytics $[1,2]$. An important group of complex carbohydrates are glycosaminoglycans (GAGs). These are strongly acidic polysaccharides with a linear sequence of repeating disaccharide units [3]. Based on the structure of these disaccharides, five families of GAGs are distinguished (Electronic Supplementary Material (ESM) Figure S1). With the exception of naturally occurring hyaluronan, the backbone is diversely sulfated, giving rise to a multitude of isomeric sequences.

GAGs are ubiquitous in the extracellular matrix and on cell surfaces [4]. Both as glycoconjugates and in unconjugated form, they mediate various physio- and pathophysiological processes, such as haemostasis, inflammation, tumorigenesis or target-cell recognition in infections $[5,6]$. Heparin - arguably the best known and pharmaceutically most relevant natural GAG-is a widely used and potent anticoagulant [7]. Due to its unfavorable pharmacokinetic properties, natural heparin is being increasingly substituted by low molecular weight heparins (LMWHs) and synthetic GAG analogues. The best example of the latter approach is fondaparinux (Arixtra®), a heparinrelated fully synthetic pentasaccharide, approved by the EMA and the FDA (Fig. 1a). 

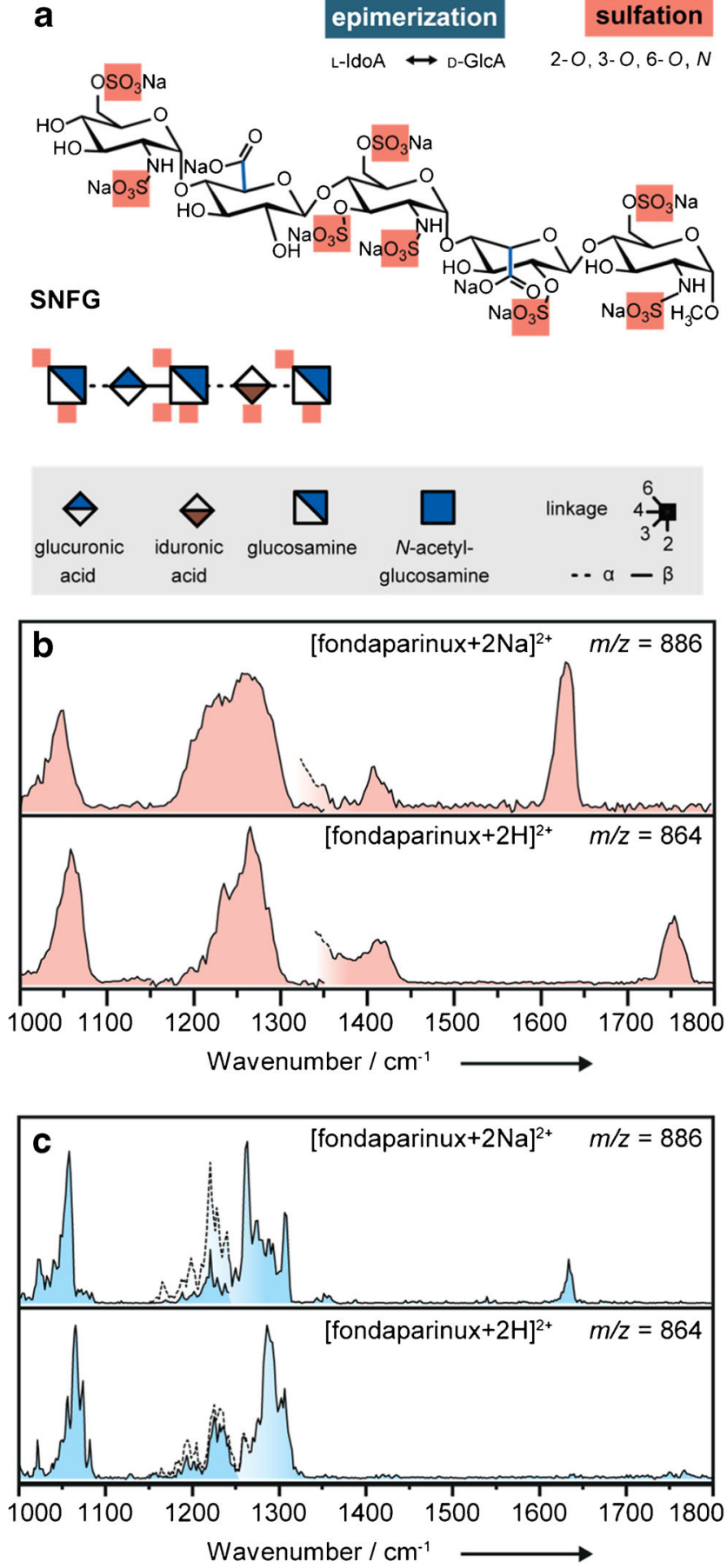

Fig. 1 a The synthetic anticoagulant fondaparinux (as sodium salt) presented as chemical structure and in the symbol nomenclature for glycans (SNFG, legend given in grey box). Highlighted in red are the positions of the sulfate groups. b IRMPD spectroscopy of fondaparinuxsodium salt (1727 Da) investigated as adduct with two additional sodium ions [fondparinux $+2 \mathrm{Na}]^{2+}$ (upper panel) and as doubly protonated species [fondaparinux $+2 \mathrm{H}]^{2+}$ (lower panel).c Cryogenic IR spectroscopy in helium nanodroplets of the aforementioned ions. Dashed lines indicate an overlap of measurements using different experimental conditions (see ESM)

Improved quality control in pharmaceutical analysis and more broadly the pursuit to elucidate the glycocode, both urge the development of efficient sequencing methods. However, the analysis of GAGs has proven to be extremely challenging. Despite the relatively simple backbone, they exhibit immense complexity, which arises from two aspects of their structure: size polydispersity and sequence microheterogeneity. As polydisperse systems, their degree of polymerization is not well-defined, making efficient separation methods essential. Sequence microheterogeneity, on the other hand, is the result of uronic acid epimerization and the extent and position of sulfation. Multidimensional workflows combining chromatographic or electrophoretic separations with mass spectrometry (MS) have been traditionally employed to tackle the aforementioned difficulties $[8,9]$. Here, the high charge density and the instantaneous, often unintended loss of sulfates during ion manipulation cause additional problems. In recent years, significant progress has been made in using novel electronbased ion activation methods (EDD [10], EID [11], NETD [12]) and ultraviolet photodissociation (UVPD) [13] to improve the capabilities of MS to overcome the unique challenges of GAG analysis. Another promising direction is to employ ion mobility spectrometry (IMS) to separate isomers or isomeric fragments prior to MS analysis [14, 15]. A complementary method to MS-based approaches is Fourier transform infrared (FTIR) spectroscopy, which has been employed for the spectroscopic profiling of intact biological samples $[16,17]$. However, despite the aforementioned successful attempts, GAGs are still largely underexplored.

The potential of gas-phase infrared (IR) spectroscopy on mass-selected ions to tackle the complexity of GAGs has been demonstrated using infrared multiple photon dissociation (IRMPD) spectroscopy [18, 19] and messenger-tagging spectroscopy [20]. Generally, spectra measured at low temperatures and without excessive ion heating proved to be better resolved. Here, we employ IRMPD spectroscopy and cryogenic IR spectroscopy in helium nanodroplets in a comparative manner to benchmark the analytical performance of each method. The aforementioned spectroscopic studies focused on disaccharides. Here, we extend the molecular space and report gas-phase IR spectra for synthetic tetra- and pentasaccharides with a different degree of sulfation. Such well-defined structures are well-suited for proof-of-principle analyses, exhibiting every aspect of sequence microheterogeneity, including uronic acid stereochemistry. In addition, the investigated molecules are similar in size to the smallest GAG oligosaccharides that carry biological information [21]. Finally, they provide residue overlap that is essential for sequencing longer GAG chains based on their characteristic fragments.

\section{Experimental methods}

Experimental details on sample preparation, IRMPD spectroscopy and cryogenic IR spectroscopy in helium nanodroplets 
can be found in the ESM. In brief, the experiments utilized in this work comprise of two types of action spectroscopy. The main difference is the type of action, namely dissociation versus ejection from a helium nanodroplet (ESM Figure S2). The former is ideal for ions with a low-energy dissociation channel to prevent spectral broadening resulting from unintended ion heating. It is well-known that highly sulfated glycosaminoglycans, both as protonated and deprotonated ions, are challenging to analyze with mass spectrometry-based techniques because of their fragility [22]. However, this fragility can be highly advantageous for a dissociation-based action spectroscopic method, such as IRMPD spectroscopy. Cryogenic IR spectroscopy in helium nanodroplets, on the other hand, is performed on ions cooled to $0.4 \mathrm{~K}$. As a result, highly resolved spectra are typically obtained, which enable the differentiation of minute structural differences in isomeric glycan ions and could potentially serve as fingerprints for identification $[23$, 24].

\section{Results and discussion}

First, the commercially available fondaparinux-sodium salt (1727 Da, Fig. 1a) was investigated as doubly protonated species [fondaparinux $+2 \mathrm{H}]^{2+}(\mathrm{m} / \mathrm{z}$ 864) and as adduct with two additional sodium ions [fondaparinux $+2 \mathrm{Na}]^{2+}(\mathrm{m} / \mathrm{z}$ 886). Using IRMPD, IR spectra for both species were recorded (Fig. 1b). Activation of both precursor ions leads to the loss of one of the eight possible sulfate groups as neutral $\mathrm{SO}_{3}$. The level of activation, i.e., the number of photons exciting the molecule, is tuned such that ideally only one loss channel is populated. Both spectra show three regions of absorptions. Most specific for the investigated ions are the positons of bands above $1600 \mathrm{~cm}^{-1}$, a region that is typically attributed to the stretching vibrations of carbonyl and carboxylate functional groups. The $[\text { fondaparinux }+2 \mathrm{H}]^{2+}$ ion exhibits a strong absorption centered at $1755 \mathrm{~cm}^{-1}$, which indicates that two protons are located at the carboxyl functional groups, making them neutral. In comparison, the sodiated analogue $[\text { fondparinux }+2 \mathrm{Na}]^{2+}$ shows a strong stretching vibration of the charged carboxylates at $1630 \mathrm{~cm}^{-1}$. Between 1200 and $1450 \mathrm{~cm}^{-1}$, mainly the antisymmetric $\mathrm{SO}_{3}{ }^{-}$stretching modes from multiple sulfate groups are observable [18], with minor contributions of weak $\mathrm{C}-\mathrm{H}$ bending vibrations. In the lower wavenumber range, combined $\mathrm{C}-\mathrm{O}$ and $\mathrm{C}-\mathrm{C}$ stretching vibrations from the glycan core and also the symmetric $\mathrm{SO}_{3}{ }^{-}$ stretching modes are typically found. Overall, the spectra are rather congested, especially in the lower wavenumber range.

To qualitatively benchmark the gain in spectral quality at low ion temperature, the IR spectra for both species $[\text { fondparinux }+2 \mathrm{Na}]^{2+}$ and [fondaparinux $\left.+2 \mathrm{H}\right]^{2+}$ were recorded using cryogenic IR spectroscopy (Fig. 1c). Generally, the position of the absorption bands obtained in IRMPD is reproduced. Yet, the spectral resolution in the cryogenic IR spectra is much higher even in the lower wavenumber range. A limitation is that the intensity of bands in the higher wavenumber range is much lower and as a consequence the carbonyl vibration above $1750 \mathrm{~cm}^{-1}$ cannot be sufficiently resolved.

As cryogenic IR spectroscopy yields spectra of improved resolution, the ability of the method to provide discrete spectral features potentially diagnostic to the sulfation pattern was tested. To do so, a model system of the hyaluronic acid (HA) family was studied. Sulfated hyaluronic acid (SHA) derivatives are in focus of current research to systematically tailor GAG-protein interactions (Fig. 2a) [25]. These newly developed potential drug candidates serve as well-defined standards in this study. With their low degree of sulfation, high stability in a mass spectrometry experiment and acidic nature, negative ion mode is well-suited for these molecules. The IR spectra for the non-sulfated HA tetrasaccharide as [HA-2H] $]^{2-}(\mathrm{m} / \mathrm{z}, 400)$
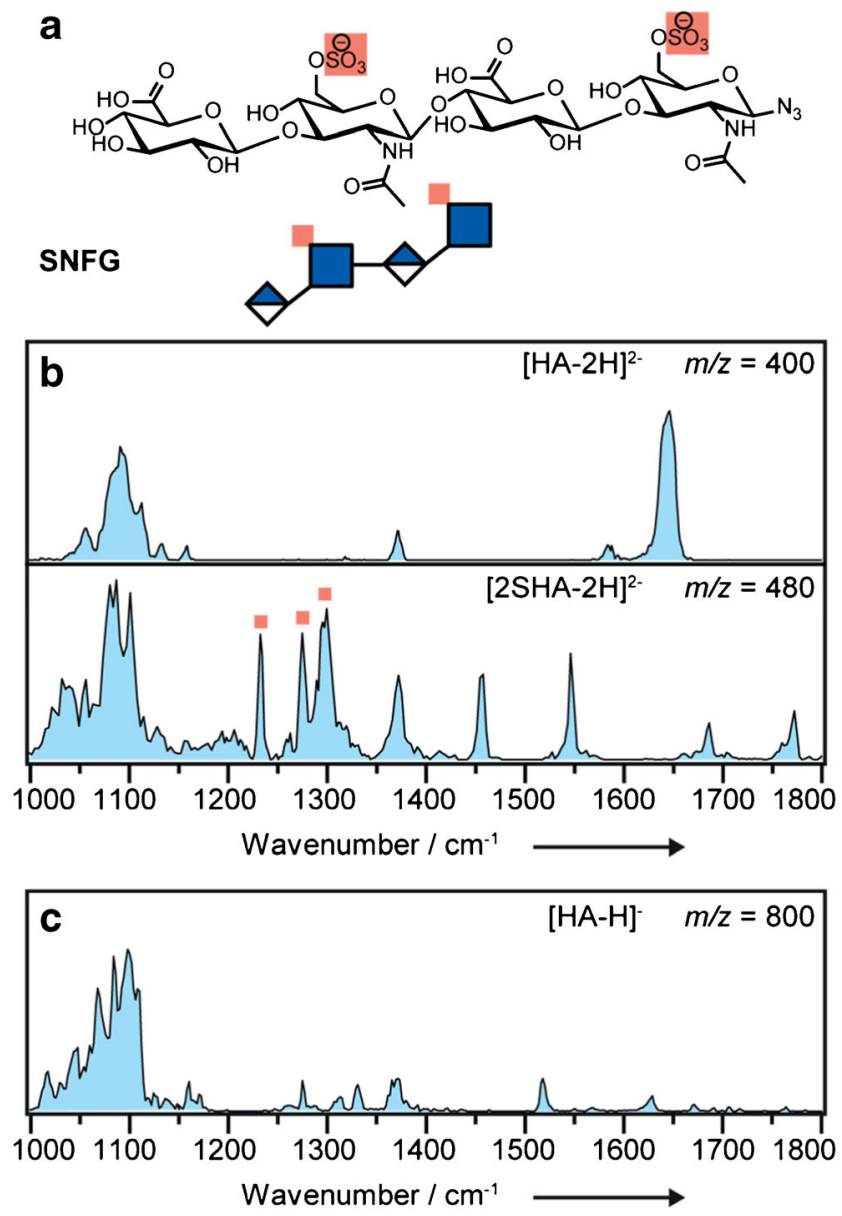

Fig. 2 a Chemical structure and SNFG representation of the investigated synthetic, sulfated (highlighted in red) hyaluronic acid (SHA) derivative. b Cryogenic IR spectroscopy in helium nanodroplets of the non-sufated hyaluronic acid (HA) as [HA-2H] $]^{2-}$ (upper panel) and the 2SHA derivative as $[2 \mathrm{SHA}-2 \mathrm{H}]^{2-}$ (lower panel). Absorption bands corresponding to sulfate groups are highlighted with red squares. c Cryogenic IR spectroscopy in helium nanodroplets of the non-sufated HA as [HA-H] 
and the doubly sulfated 2SHA derivative as [2SHA-2H $]^{2-}$ $(\mathrm{m} / \mathrm{z}, 480)$ were recorded using cryogenic IR spectroscopy (Fig. 2b). The strongest absorption in the spectrum of the non-sulfated $[\mathrm{HA}-2 \mathrm{H}]^{2-}$ is the carboxylate antisymmetric stretching vibration at $1645 \mathrm{~cm}^{-1}$. The presence of this band confirms the deprotonation of the carboxyl functional group. The amide $\mathrm{I}$ band (the $\mathrm{C}=\mathrm{O}$ stretching vibration of secondary amides) arising from the GlcNAc moiety is most likely overlapped by the strong vibration of the carboxylate anion. The corresponding amide II and III vibrations are assigned to the features observed at $1585 \mathrm{~cm}^{-1}$ and $1372 \mathrm{~cm}^{-1}$, respectively. Absorptions between 1000 and $1200 \mathrm{~cm}^{-1}$ mainly stem from the glycan core.

In order to elucidate the impact of sulfation, the spectrum of the doubly sulfated, but otherwise chemically identical glycan $[2 \mathrm{SHA}-2 \mathrm{H}]^{2-}$ was recorded for comparison. As expected from the relative proton affinities of the carboxylate and sulfate moieties, the carboxyl functional groups are neutral, which is confirmed by the $\mathrm{C}=\mathrm{O}$ stretching vibration at $1770 \mathrm{~cm}^{-1}$. The signal at $1685 \mathrm{~cm}^{-1}$ is assigned to the amide I vibrations. The amide II and III vibrations can be assigned qualitatively to the bands at 1547 and $1372 \mathrm{~cm}^{-1}$. The vibration at $1455 \mathrm{~cm}^{-1}$ is in a spectral region in which $\mathrm{O}-\mathrm{H}$ bending modes in carboxyl groups are typically found. Most importantly, the sulfates show multiple well-resolved bands between 1200 and $1350 \mathrm{~cm}^{-1}$ (Fig. 2b, lower panel, highlighted with red squares).

Finding the optimal charge state is crucial to obtaining good spectral quality. With two carboxylate functional groups, the singly deprotonated ion of the tetrasaccharide HA yields a congested spectrum (Fig. 2c). Two chemically almost identical deprotonation sites aid the formation of two deprotomers, each with a multitude of individual conformers. In addition, the dense hydrogen bonding network within the molecule can promote charge migration, which further increases the number of potentially observable species [26].

\section{Conclusion}

In summary, we here demonstrate the potential of IR action spectroscopy in the range from 1000 to $1800 \mathrm{~cm}^{-1}$ for the structural characterization of highly complex GAG oligosaccharides. Well-resolved IR spectra of oligosaccharides up to pentasaccharides were obtained using cryogenic IR spectroscopy in helium nanodroplets. Signals arising from sulfate groups appear in a spectral range in which typically no other diagnostic vibrations occur. As a result, vibrational patterns with high informational content are highly resolved. The optimal charge state depends on the functional groups present and is crucial for the spectral quality. Besides being sensitive to minute structural differences, cryogenic IR spectroscopy on mass-selected ions has the potential to be implemented in existing MS-based workflows. Current MS-based databases [27] could be extended with IR fingerprints of intact and fragment ions of GAGs. As such, gas-phase IR spectroscopy could serve as a key analytical technique for the characterization of GAG oligosaccharides in the future.

Acknowledgements Open access funding provided by Max Planck Society. The authors gratefully acknowledge generous funding by the Freie Universität Berlin, the Max Planck Society and the great expertise of Sandy Gewinner and Dr. Wieland Schöllkopf of the FHI free electron laser. DAT acknowledges support from the Alexander von Humboldt Foundation.

Funding information This research is funded by the Deutsche Forschungsgemeinschaft (DFG, German Research Foundation) Projektnummer 372486779 - SFB 1340.

\section{Compliance with ethical standards}

Conflict of interest The authors declare that they have no competing interests.

Open Access This article is licensed under a Creative Commons Attribution 4.0 International License, which permits use, sharing, adaptation, distribution and reproduction in any medium or format, as long as you give appropriate credit to the original author(s) and the source, provide a link to the Creative Commons licence, and indicate if changes were made. The images or other third party material in this article are included in the article's Creative Commons licence, unless indicated otherwise in a credit line to the material. If material is not included in the article's Creative Commons licence and your intended use is not permitted by statutory regulation or exceeds the permitted use, you will need to obtain permission directly from the copyright holder. To view a copy of this licence, visit http://creativecommons.org/licenses/by/4.0/.

\section{References}

1. Gray CJ, Migas LG, Barran PE, Pagel K, Seeberger PH, Eyers CE et al. Advancing solutions to the carbohydrate sequencing challenge. J Am Chem Soc. 2019.

2. Mucha E, Stuckmann A, Marianski M, Struwe WB, Meijer G, Pagel K. In-depth structural analysis of glycans in the gas phase. Chem Sci. 2019.

3. Gandhi NS, Mancera RL. The structure of Glycosaminoglycans and their interactions with proteins. Chem Biol Drug Des. 2008;72(6):455-82.

4. Lindahl U, Couchman J, Kimata K, Esko JD. Proteoglycans and sulfated glycosaminoglycans. In: Varki A, Cummings RD, Esko JD, Stanley P, Hart GW, Aebi M et al., editors. Essentials of glycobiology 3ed.: Cold Spring Harbor (NY): Cold Spring Harbor Laboratory Press; 2017.

5. Kamhi E, Joo EJ, Dordick JS, Linhardt RJ. Glycosaminoglycans in infectious disease. Biol Rev. 2013;88(4):928-43.

6. Sasisekharan R, Shriver Z, Venkataraman G, Narayanasami U. Roles of heparan-sulphate glycosaminoglycans in cancer. Nat Rev Cancer. 2002;2(7):521-8.

7. Capila I, Linhardt RJ. Heparin-protein interactions. Angew Chem Int Ed. 2002;41(3):390-412. 
8. Jones CJ, Beni S, Limtiaco JFK, Langeslay DJ, Larive CK. Heparin characterization: challenges and solutions. Ann Rev Anal Chem. 2011;4(1):439-65.

9. Ly M, Leach FE, Laremore TN, Toida T, Amster IJ, Linhardt RJ. The proteoglycan bikunin has a defined sequence. Nat Chem Biol. 2011;7(11):827-33.

10. Wolff JJ, Amster IJ, Chi L, Linhardt RJ. Electron detachment dissociation of glycosaminoglycan tetrasaccharides. J Am Soc Mass Spectrom. 2007;18(2):234-44.

11. Wolff JJ, Laremore TN, Aslam H, Linhardt RJ, Amster IJ. Electroninduced dissociation of glycosaminoglycan tetrasaccharides. J Am Soc Mass Spectrom. 2008;19(10):1449-58.

12. Leach FE, Riley NM, Westphall MS, Coon JJ, Amster IJ. Negative electron transfer dissociation sequencing of increasingly sulfated glycosaminoglycan oligosaccharides on an Orbitrap mass spectrometer. J Am Soc Mass Spectrom. 2017;28(9):1844-54.

13. Klein DR, Leach FE, Amster IJ, Brodbelt JS. Structural characterization of glycosaminoglycan carbohydrates using ultraviolet photodissociation. Anal Chem. 2019;91(9):6019-26.

14. Wei J, Wu J, Tang Y, Ridgeway ME, Park MA, Costello CE, et al. Characterization and quantification of highly sulfated glycosaminoglycan isomers by gated-trapped ion mobility spectrometry negative electron transfer dissociation MS/MS. Anal Chem. 2019;91(4): 2994-3001.

15. Seo Y, Andaya A, Leary JA. Preparation, separation, and conformational analysis of differentially sulfated heparin octasaccharide isomers using ion mobility mass spectrometry. Anal Chem. 2012;84(5):2416-23.

16. Brézillon S, Untereiner V, Lovergne L, Tadeo I, Noguera R, Maquart F-X, et al. Glycosaminoglycan profiling in different cell types using infrared spectroscopy and imaging. Anal Bioanal Chem. 2014;406(24):5795-803.

17. Mohamed HT, Untereiner V, Sockalingum GD, Brézillon S. Implementation of infrared and Raman modalities for glycosaminoglycan characterization in complex systems. Glycoconj J. 2017;34(3):309-23.

18. Schindler B, Barnes L, Gray CJ, Chambert S, Flitsch SL, Oomens J, et al. IRMPD spectroscopy sheds new (infrared) light on the sulfate pattern of carbohydrates. J Phys Chem A. 2017;121(10):2114-20.
19. Renois-Predelus G, Schindler B, Compagnon I. Analysis of sulfate patterns in glycosaminoglycan oligosaccharides by MSn coupled to infrared ion spectroscopy: the case of GalNAc4S and GalNAc6S. J Am Soc Mass Spectrom. 2018;29(6):1242-9.

20. Khanal N, Masellis C, Kamrath MZ, Clemmer DE, Rizzo TR. Glycosaminoglycan analysis by cryogenic messenger-tagging IR spectroscopy combined with IMS-MS. Anal Chem. 2017;89(14): 7601-6.

21. Gama CI, Tully SE, Sotogaku N, Clark PM, Rawat M, Vaidehi N, et al. Sulfation patterns of glycosaminoglycans encode molecular recognition and activity. Nat Chem Biol. 2006;2(9):467-73.

22. Kailemia MJ, Li L, Ly M, Linhardt RJ, Amster IJ. Complete mass spectral characterization of a synthetic ultralow-molecular-weight heparin using collision-induced dissociation. Anal Chem. 2012;84(13):5475-8.

23. Mucha E, Gonzalez Florez AI, Marianski M, Thomas DA, Hoffmann W, Struwe WB, et al. Glycan fingerprinting via coldion infrared spectroscopy. Angew Chem Int Ed. 2017;56(37): $11248-51$.

24. Mucha E, Lettow M, Marianski M, Thomas DA, Struwe WB, Harvey DJ, et al. Fucose migration in intact protonated glycan ions: a universal phenomenon in mass spectrometry. Angew Chem Int Ed. 2018;57(25):7440-3.

25. Köhling S, Blaszkiewicz J, Ruiz-Gómez G, Fernández-Bachiller MI, Lemmnitzer K, Panitz N, et al. Syntheses of defined sulfated oligohyaluronans reveal structural effects, diversity and thermodynamics of GAG-protein binding. Chem Sci. 2019;10(3):866-78.

26. Struwe WB, Baldauf C, Hofmann J, Rudd PM, Pagel K. Ion mobility separation of deprotonated oligosaccharide isomers - evidence for gas-phase charge migration. Chem Commun. 2016;52(83):12353-6.

27. Hogan JD, Klein JA, Wu J, Chopra P, Boons G-J, Carvalho L, et al. Software for peak finding and elemental composition assignment for glycosaminoglycan tandem mass spectra. Mol Cell Proteomics. 2018;17(7):1448-56.

Publisher's note Springer Nature remains neutral with regard to jurisdictional claims in published maps and institutional affiliations. 